Recibido: marzo de 2018

Aprobado: mayo de 2018

DOI: https://doi.org/10.15332/rev.m.v15i0.2176

Strade di Guane, Santander (Colombia) Fonte: Autori, 2013

\section{LA CHIESA DI SANTA LUCIA DI GUANE - BARICHARA: ANALISI STRUTTURALE PER LA SUA SALVAGUARDIA*}

\author{
Michele Paradiso ** - Università degli Studi di Firenze, Italia \\ Chiara Bini $^{* * *}$ - Italia \\ Natascia Crescenzi ${ }^{* * * *}$ - Italia \\ Carlos Humberto Gomez Arciniegas ***** - Universidad Santo Tomás, Colombia
}

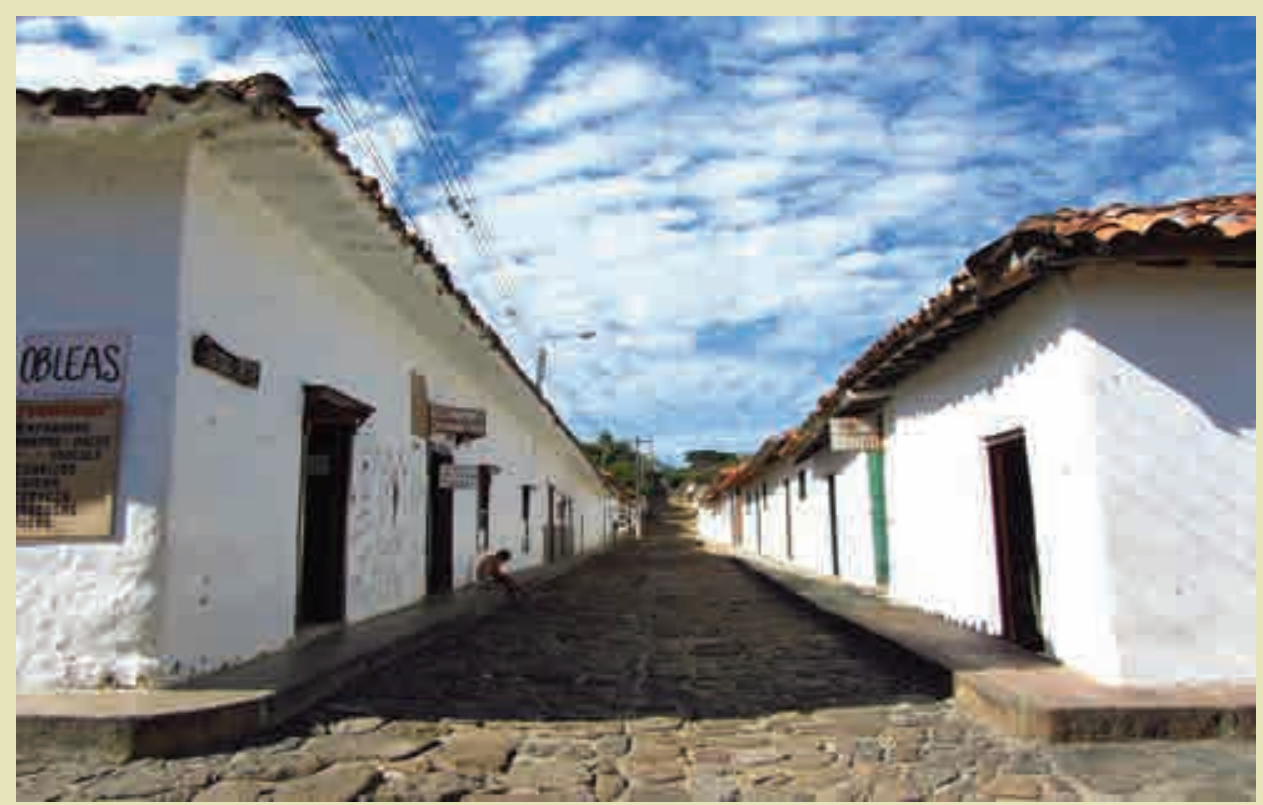

\section{RIASSUNTO}

L'articolo si riferisce allo stato di degrado strutturale della Chiesa di Santa Lucia, in Guane, Comune di Barichara, nel Dipartimento di Santander (Colombia). Lo studio fu effettuato negli anni 20I2-20I 3 in collaborazione con la Facoltà di Architettura dell'Universidad Santo Tomás, sede Bucaramanga. E' stata applicata la metodologia tipica indicata dalle Carte Internazionali del Restauro di ICOMOS-UNESCO. II quadro fessurativo, complesso e preoccupante, che presentava la Chiesa, Monumento Nazionale, alla data della presa in carico del lavoro, é conseguente al cedimento verticale delle fondazioni, peraltro molto scarse, dell'angolata destra in facciata principale. Lo studio che qui si presenta indica le soluzioni di messa in sicurezza dell'edificio e le ipotesi di massima per un suo consolidamento, con tecniche non invasive. L'occasione di questo racconto é per gli autori, anche l'occasione per riflettere sulle dinamiche di gestione a livello nazionale, della conservazione del patrimono storico costruito. Dinamiche che appaiono lente, estremamente burocratizzate e accompagnate da scarso dialogo tra le istituzioni preposte alla salvaguardia del bene storico. Il lavoro si é poi concretizzato, per le due coautrici, nella loro tesi di laurea in architettura, discussa presso la Facoltà di Architettura dell’Università degli Studi di Firenze nell'a.a. 20I2-2013.

\section{PAROLE CHIAVE}

Conservazione, Guane, meccanismi di collasso, restauro, cedimento del suolo. 


\section{LA IGLESIA DE SANTA LUCÍA DE GUANE - BARICHARA: ANÁLISIS ESTRUCTURAL PARA SU SALVAGUARDIA}

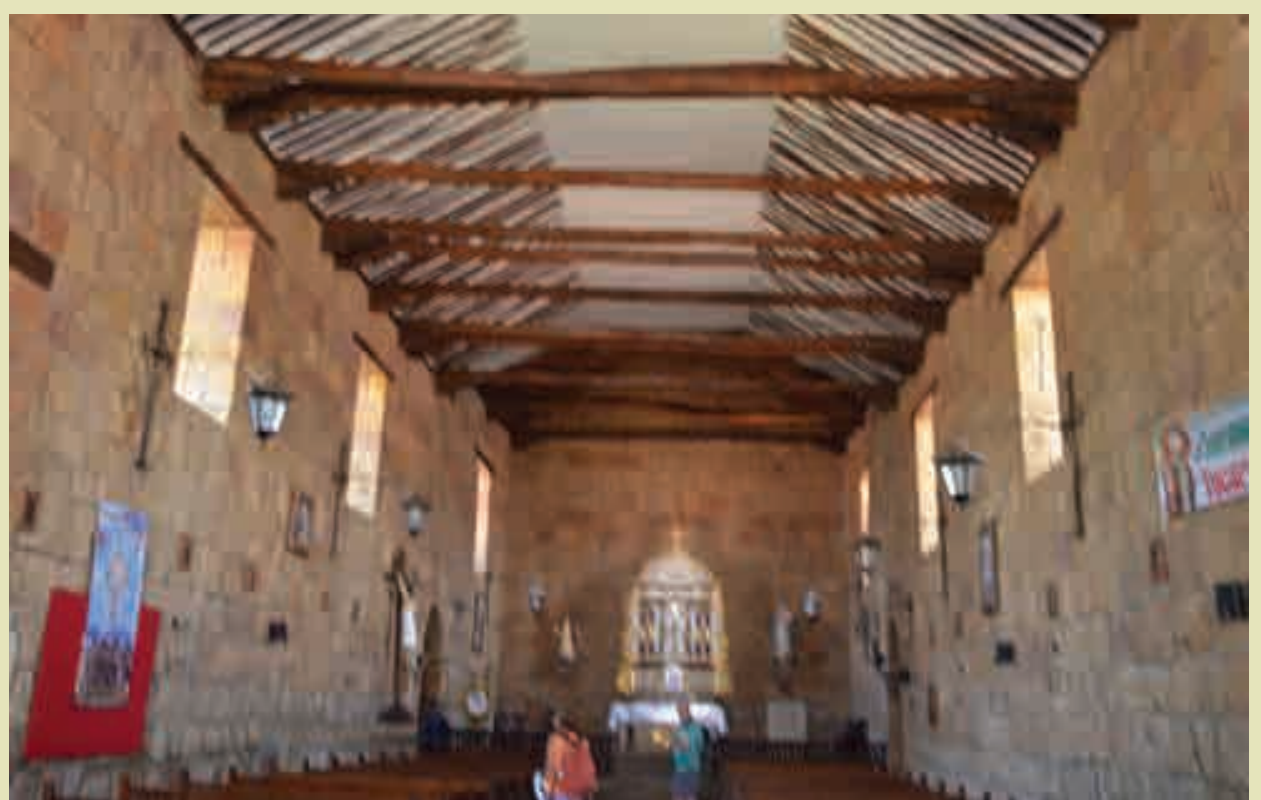

\section{RESUMEN}

El artículo se refiere al estado de deterioro estructural de la iglesia de Santa Lucía, en Guane, muncicipio de Barichara, en el departamento de Santander (Colombia). El estudio se llevó a cabo en los años 20I2-2013 en colaboración con la Facultad de Arquitectura de la Universidad Santo Tomás Seccional Bucaramanga. Se aplicó la metodología típica indicada por las “Cartas Internacionales de la Restauración ICOMOS_UNESCO”. El conjunto de grietas, complejo y preocupante, que sufre la iglesia, Monumento Nacional, a la fecha de hacerse cargo del estudio, es como consecuencia de la subsidencia vertical de las cimentaciones, que son muy escasas, empezando por la esquina derecha de la fachada principal. El estudio presentado aquí indica las soluciones para poner en seguridad estructural al edificio y las hipótesis para una primera idea de consolidación estructural, utilizando técnicas no invasivas. Este estudio es también para los autores una oportunidad para reflexionar sobre la dinámica de la gestión, a nivel nacional, de la conservación del patrimonio histórico construido. Dinámicas que parecen lentas, extremadamente burocráticas y acompañadas por un diálogo deficiente entre las instituciones responsables de salvaguardar el patrimonio histórico. La investigación se materializó en cierta manera gracias al trabajo de las dos coautoras, en su tesis de grado en Arquitectura, en la Facultad de Arquitectura de la Universidad de Florencia en 20I2-20 I3.

\section{PALABRAS CLAVE}

Conservación, Guane, mecanismos de colapso, restauración, subsidencia del suelo.
Interior de la Iglesia de Santa Lucia. Guane, Santander (Colombia)

Fonte: Autori, 2013.
**** Arquitecta por la Universidad de los Estudios de Florencia (Italia). Su trabajo de grado lo realizó sobre el estado estructural y de conservación de la Iglesia de Santa Lucía, en Guane (Santander). El trabajo de campo allí realizado le sirvió para adquirir mayor experiencia en la restauración y conservación de edificios históricos. Actualmente trabaja en la Asociación Cultural Controchiave en Roma. Correo electrónico: natascia. crescenzi@stud.unifi.it

***** Arquitecto por la Universidad Santo Tomás Bucaramanga (Colombia); especialista en Planificación del Territorio para el Desarrollo - Politécnico di Milano (Italia) - Université de Paris I, Pantheon-Sorbonne, París (Francia) MSc en Relaciones Internacionales y Estudios Estratégicos con énfasis en Políticas de Desarrollo - Lancaster Uinversity (Inglaterra), y PhD en Planificación Urbana, Territorial y Ambiental - Politécnico di Milano (Italia). Docente investigador, líder de la línea de investigación en Planificación y Gestión del Territorio, adscrita al grupo de Investigaciones de la Facultad de Arquitectura de la Universidad Santo Tomás Bucaramanga (Colombia). Correo electrónico: carlos. gomez@ustabuca.edu.co 


\section{INTRODUZIONE}

Nel novembre del 20I I il Prof. Michele Paradiso, docente del Dipartimento di Architettura dell'Università di Firenze - Italia e referente dell'accordo di collaborazione interuniversitaria con la Facoltà di Architettura della Universidad Santo Tomàs di Bucaramanga, fu invitato dai direttivi locali a trascorre una settimana di studio a Bucaramanga. Una delle prime occasioni per conoscere il patrimonio storico di Santander, fu la visita a Barichara, e poi al paesino di Guane, visita condivisa con l'allora Preside della Facoltà di Architettura, Architetta Claudia Patricia Uribe Rodriguez. Era interesse della Decana coinvolgere il Dipartimento fiorentno, in una collaborazione internazionale volta alla salvaguardia sia della Chiesa di Santa Lucia, sia dell'intero poblado. Con i docenti colombiani, componenti il gruppo di studio, denominato Linea de Patrimonio, ci si sarebbe occupati di preparare la documentazione necessaria affinché venisse elevata al rango di Monumento Nacional, non solo la Chiesa, che già lo era, ma tutto il paesino. La Chiesa di Guane però soffriva di un importante quadro fessurativo, che ne miacciava la stabilità stitica, particolarmente nella zona della capella del Niño Jesús, a destra dell'ingresso. Profonde lesioni evidenziavano già il distacco della cappella dal corpo di fabbrica principale ed era urgente mettere mano ad uno studio approfondito. Questo fu assunto in carico dalla componente fiorentina, mentre la componente docente locale, corroborata da propri studenti, si sarebbe occupata degli aspetti urbanistici, architettonici e ambientali di Guane. Nel gennaio del 2012 arrivò la notizia che una forte pioggia aveva fatto crollare il tetto ligneo della cappella Niño Jesús, mettendo in serie ambasce il Parroco. Fu così che nel maggio del 2012 un gruppo di nove studenti fiorentini e il docente succitato, sbarcarono a Guane per iniziare gli studi. Natascia Crescenzi e Chiara Bini decisero che il tema della Chiesa di Santa Lucia di Guane sarebbe stato oggetto della loro tesi di laurea in Architettura. E rimasero a Guane più di tre mesi. Quello che segue è un documento il cui fulcro principale e il risultato dei loro studi e del supporto degli accademici preocupati per la salvaguarda del patrimonio di Santander.

\section{ASPETTI STORICI, URBANISTICI E ARCHITETTONICI}

La chiesa di Santa Lucia a Guane nasce come iglesia doctrinal con funzione di centro di colonizzazione, tramite la religione cattolica, del popolo indigenonativo dell'area centrale del Santander. Quest'opera fu molto ambiziosa se si paragona alle dimensioni ridotte dell'attuale villaggio di Guane, costituito da una decina di cuadras e poche centinaia di abitanti (Arenas, 2004). Non ci sorprende, invece, se pensiamo che tra i secoli XVI e XVIII Guane, o meglio Moncora, come veniva chiamato allora, era uno dei principali pueblos de indios fondati dai conquistatori spagnoli. Gli spagnoli crearono forzatamente centri di congregazione di etnie aborigene differenti andando a distruggere completamente la cultura urbana, gli usi e i costumi autoctoni e imponendo abitudini culturali e alimentari europee (lbañez, 2004). Gli indigeni vivevano di pesca e costruivano le loro case sui margini dei fiumi, non erano abituati a vivere in centri urbani densi “... no era costumbre de los aborígenes vivir agrupados en núcleos urbanos...” (Ardila, 2005).

I pueblos venivano fondati a partire dalla piazza centrale nella quale si ergeva una croce, a seguire si tracciavano le cuadras, isolati che rispondevano al rigido disegno del cardo e decumano. Analizzando la distribuzione degli abitanti nella matrice geometrica ci si poteva fare un'idea dello status sociale delle famiglie che la occupavano: le case costruite intorno alla piazza principale appartenevano ad abitanti di prestigio, spesso con incarichi pubblici, via via che ci si allontanava dal centro scendeva il ceto sociale del quale si faceva parte (Ribero, 1986). Nonostante Guane e Barichara siano stati fondate su una topografia che presenta una notevole pendenza, la loro trama urbana segue perfettamente la rigida maglia ortogonale non preoccupandosi delle caratteristiche morfologiche naturali del suolo (Fig. I, 2). 

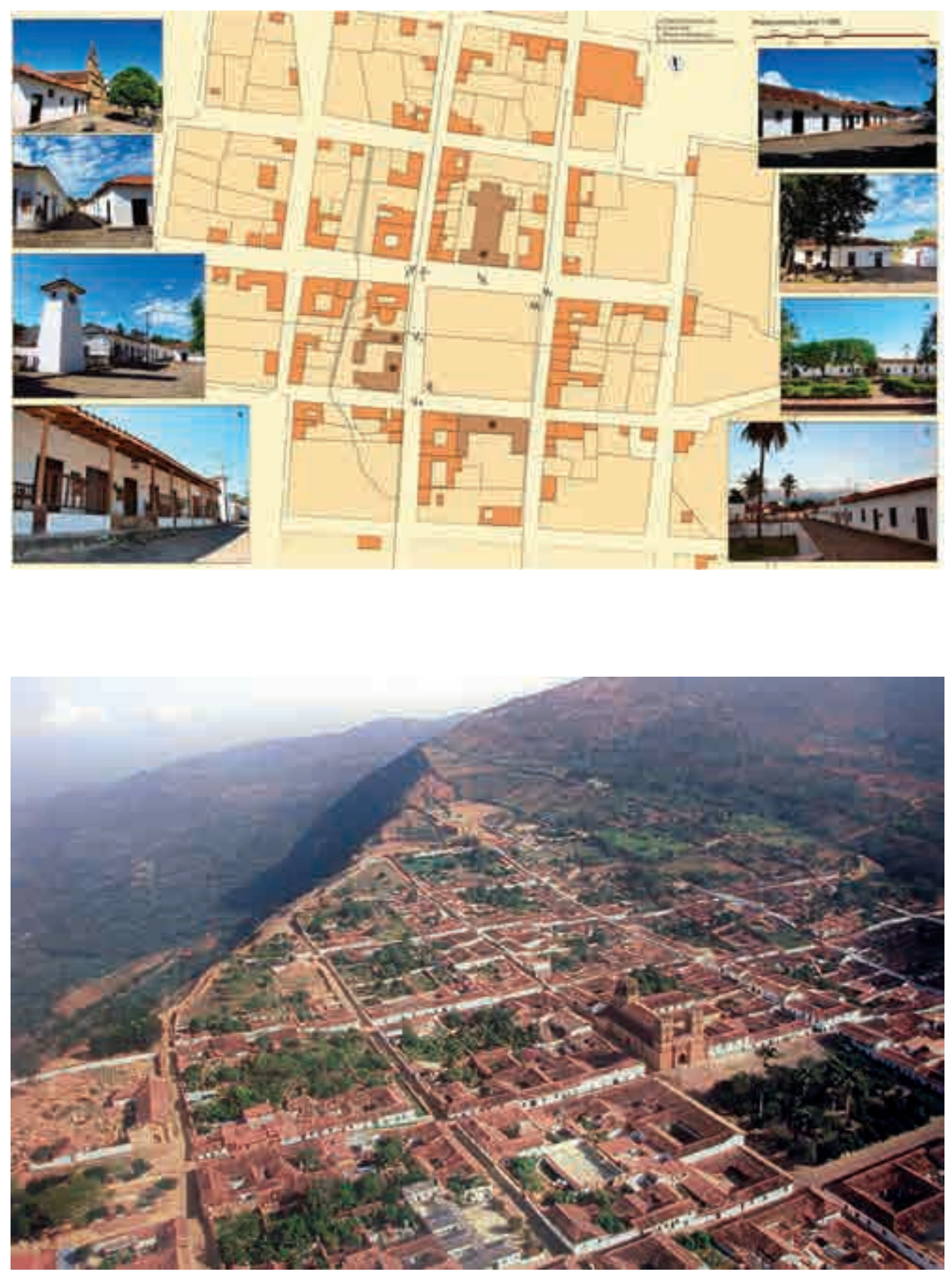

Il paese di Guane, insieme alla vicina cittadina di Barichara, si è mantenuto quasi inalterato nel tempo grazie al suo isolamento dalle principali vie di comunicazione. Qui possiamo ancora trovare gli elementi tipici dell'architettura coloniale colombiana:l'alto marciapiede in pietra, basamento che dava importanza estetica alla costruzione e nello stesso tempo riparava dalle acque meteoriche che scorrevano nelle strade non essendoci un sistema di fognatura; lo zoccolo dipinto, parte bassa delle murature probabilmente anche questo a protezione della pioggia per evitare il dilavamento dell'intonaco bianco in calce e paglia ed infine la copertura in bambù e tegole che non presentava elementi di raccolta delle acque, ma la funzione di allontanamento di queste era svolta dalla morfologia con falda a doppia pendenza (Fig. 3) (Silvarangel, 200I).
Figure I. Impianto urbano del poblado de Guane Fonte: Elaborazione grafica degli autori.
Figure 2. Foto aerea della città di Barichara Fonte: Google Earth, 2018. 
Figure 3. Schizzo di una tipica stradina di Guane Fonte: Elaborazione grafica degli autori, 2013.

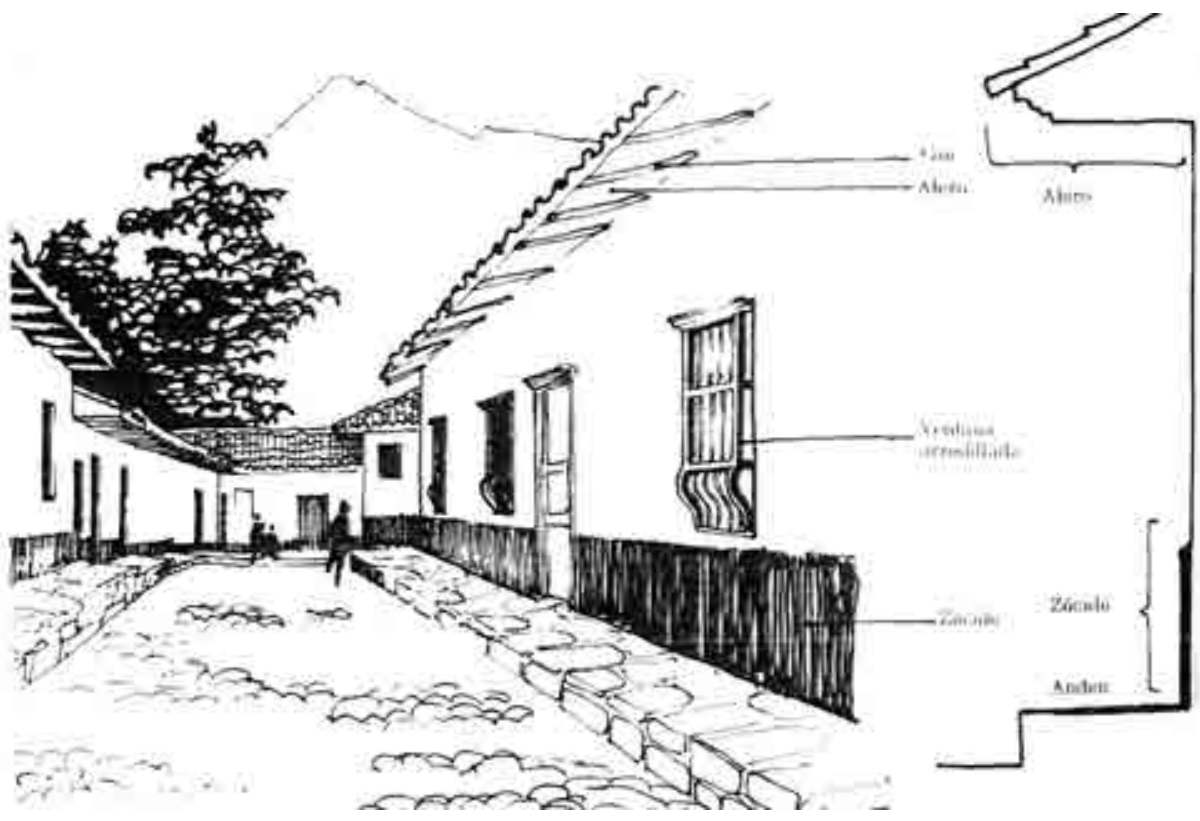

Fu Padre Alonso Ortíz Galeano agli inizi del 1600 ad occuparsi della costruzione della prima chiesa di cui si hanno testimonianze, ubicata a lato della casa cural dove sorge l'attuale museo della civiltà Guane. L'edificio si presentava "de paja y bahareque de barro y caña, de tres naves y estantillos, con dos ventanas de verjas de madera y otras de verjas de cañas" (Arenas, 2004). Nel secolo successivo Padre Francisco Basilio de Benavides, intellettuale con titolo di dottore conseguito all'Università di Santa Fe, contribuì in maniera decisiva nel dotare Guane di una nuova chiesa, sempre nello stesso sito della prima, con copertura in travi di legno, tegole e pareti intonacate di bianco "poniendoyo de mi parte todaslasvigas, toda la taxa, la cal para elblanqueado y muchas pietra labradas para elpretil de que se circunvaló" (Arenas, 2004).

Nel I78I iniziarono i lavori di edificazione dell'attuale templo che aveva cambiato ubicazione rispetto ai precedenti, erigendosi al centro del lotto sud della grande piazza centrale del paese, occupandone quasi tutta la quadra. La particolarità della sua ubicazione è legata probabilmente, oltre a ragioni culturali, alla presenza di un dislivello che permette, così, alla chiesa di dominare dall'alto l'intero paese. Nel I8I6 ci sono testimonianze del completamento dell'opera nella sua obra negra, cioè nei suoi elementi principali. Sinteticamente, queste le tappe principali (Pbro. Carreño, 20I0):

I. 1602: ricostruzione della cappella innalzata dai primi missionari

2. I76I e I778: l'edificio viene descritto nelle cronache storiche come "edificio debil, ruinoso, y de corta extencion"

3. 178I: viene concesso il permesso per la costruzione di una nuova chiesa

4. 1786: iniziano i lavori di edificazione della chiesa di Santa Lucia

5. 1874: prima messa in sicurezza (campanile a vela)

6. 1960: primo restauro

7. 1970: vengono notate le prime lesioni 


\section{RILEVAMENTO METRICO DELL'IMPIANTO ARCHITETTO- NICO E INDIVIDUAZIONE DELLE PATOLOGIE DI DEGRADO MATERICO E STRUTTURALE}

Per impostare e organizzare correttamente le fasi di rilievo della chiesa che hanno preceduto l'analisi del degrado materico e strutturale portando alla formulazione di una ipotesi di consolidamento, è stata indispensabile una prima ricognizione del luogo, correlata da un'accurata campagna fotografica e da considerazioni scritte e grafiche che permettessero di comprendere meglio le problematiche e la complessità dell'intervento (Francesci, Germani, 20I2).

A questo riguardo, il 27 maggio 2012, è stato effettuato un sopralluogo con l'allora parroco di Guane Padre Daniel Carreño Sarmiento, la Preside di allora della Facoltà di Architettura dell'Universidad Santo Tomás di Bucaramanga, Arch. Claudia Uribe, con i docenti Architetti Carlos Gómez Arciniegas, Nahir Pabón Castro, Eneida Abreu Plata, Maria Fernanda Reyes Rodríguez, oltre che al Prof. Arch. Michele Paradiso, visiting professor alla USTABUCA del Dipartimento di Architettura dell'Università degli Studi di Firenze. II sopralluogo ha permesso la raccolta di informazioni di base sulle fasi costruttive della chiesa e sui principali problemi manifestatisi fino a quel momento.

II rilevamento metrico è proceduto tramite la metodologia tradizionale, iniziando dal tracciamento del piano ideale orizzontale di riferimento, sia all'esterno che all'interno dell'edificio, in maniera da poter svolgere un rilievo georiferito del terreno e della chiesa. La seconda operazione eseguita è stata quella di definizione della pianta dell'edificio rendendola misurabile attraverso l'operazione di trilaterazione, interna ed esterna, a quota del piano di riferimento. Le misure raccolte durante la campagna di rilievo in loco sono state successivamente riportate in AutoCad, ottenendo così la pianta interna es esterna della navata centrale e delle quattro cappelle inoltre allo spessore dell'apparato murario. Una volta disegnata la pianta si è proceduto con le coltellazioni verticali, verso il basso per definire l'andamento del pavimento e del terreno e verso l'alto per definire l'altezza dell'edificio. È stata posta particolare attenzione all'identificazione di eventuali fuori piombo delle pareti, sia interni che esterni. Eccezione al metodo è stata fatta per il rilevamento del campanile a vela, il quale, essendo di difficile accessibilità è stato calcolato attraverso il rilievo fotografico (Fig. 4).

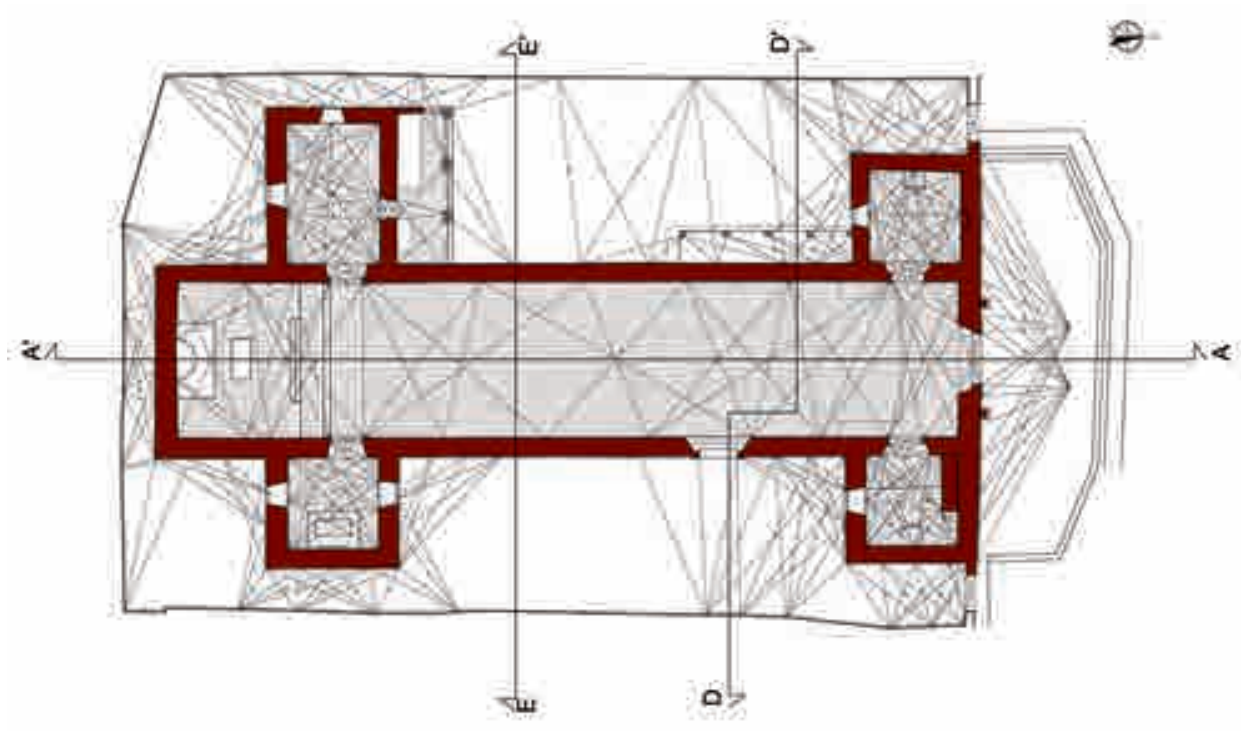

Figure 4. Pianta della Chiesa, trilaterazioni Fonte: Elaborazione grafica degli autori. 
È stata individuata, di seguito al rilievo, la stratigrafia delle fasi costruttive attraverso l'ausilio della documentazione storica, oltre che all'osservazione diretta, evidenziando le fasi di modificazione dell'edificio con gli interventi di sostituzione o sovrapposizione succedutisi nel tempo insieme alle diversità formali che ne sono conseguite. L'edificio si presenta come un enorme parallelepipedo rettangolare al quale si aggiungono quattro annessi, molto probabilmente di epoche più recenti rispetto al corpo centrale.L'entrata alla navata avviene tramite il portone che si apre sulla piazza, ma è presente anche un'altra entrata situata lateralmente sul fronte est. La nave presenta una dimensione di circa $9 \mathrm{~m}$ x $45 \mathrm{~m}$, con un'altezza di I I m; nella parte absidale si nota un innalzamento del livello del piano di calpestio ed anche dell'altezza della copertura, apprezzabile anche dall'esterno attraverso la relazione dei volumi.La facciata prospicente l'ampio sagrato ha un fronte simmetrico con un'esile campanile a vela. L'entrata presenta un arco a tutto sesto leggermente rialzato sormontato da una bifora, che illumina il coro, non perfettamente in asse con il centro dell'arco sottostante.

A destra della facciatasi trova la cappella del Niño Jesús di pianta quadrangolare di circa 5,4 m x 5,4 m. Risulta la parte della chiesa più vulnerabile. La cappella della Niña María si trova sul lato sinistro della nave specularmente a quella del Niño Jesús e porta attraverso una scala ad accedere al coro. La Sagrestia si apre sul lato destro della navata in corrispondenza del presbiterio e presenta un passaggio alla ronda, la parte esterna, tramite un piccolo portico con colonne in stile neoclassico.Infine la cappella della Santa Lucia, cui è dedicata la Chiesa, è ubicata simmetricamente rispetto alla Sagrestia (Figure $5,6,7,8,9,10)$.

L'apparecchiatura muraria della nave e delle cappelle ha uno spessore medio di $120 \mathrm{~cm}$ ed è realizzata a sacco con il confinamento verticale in pietra caliza, calcarenite lavorata in grossi blocchi, e il riempimento in inerti e materiale di risulta. La struttura della muratura a causa della sua composizione e apparecchiatura non ha mai un comportamento monolitico; essa infatti tende ad essere tripartita a causa della malta di riempimento del sacco che non riesce a dare un sufficiente legame diatonico con i paramenti esterni. Inoltre dal punto di vista statico il materiale inerte all'interno delle pareti non ha nessuna resistenza strutturale e la capacità portante viene del tutto svolta dalle due folii laterali in pietra (Cigni, 1978). La copertura a capanna della navata conserva lo stile più antico, con travi di bordo, arcarecci e cielo raso, controsoffitto. La stessa è resa non spingente, o perlomeno tenta di esserlo, attraverso l'uso di tiranti di piano in legno. Le coperture dei quattro corpi laterali sono a semplice falda inclinata o a doppia falda. Tutti gli elementi in legno sono appena sbozzati e alcuni tiranti di piano appaiono come veri e propri tronchi dal profilo irregolare, appena trattati e posti in opera. Ciò rende l'atmosfera particolarmente suggestiva. La tecnica utilizzata per la costruzione della copertura è chiamata par y nudillo, e consta dell'uso di coppie di travi che vengono disposte secondo la pendenza del tetto, unite superiormente da una trave di colmo chiamata hilera o cumbrera, e inferiormente incastrate ad un'altra trave in legno chiamata solera, che a sua volta è tenuta ferma da un'ulteriore trave che svolge la funzione di tirante, denominata estribo. Posta in opera questa armatura principale, si possono montare i piccoli tiranti orizzontali chiamati nudillos, che nel nostro caso vanno a costituire la struttura del cielo raso. Questo tipo di struttura assicura una maggiore resistenza della copertura, dovuta alla rigidezza che apporta a tutto il sistema l'elemento del nudillo (Ciaccia, 2003). I problemi e la vulnerabilità di questo tipo di coperture sono dovuti alle vulnerabilità meccaniche del legno, non perfetta messa in opera dell'orditura e alle dimensioni degli stessi elementi, caratteristiche, queste, che possono creare problemi di precario equilibrio statico e portare al collasso la struttura (Mastrodicasa, 1974). 

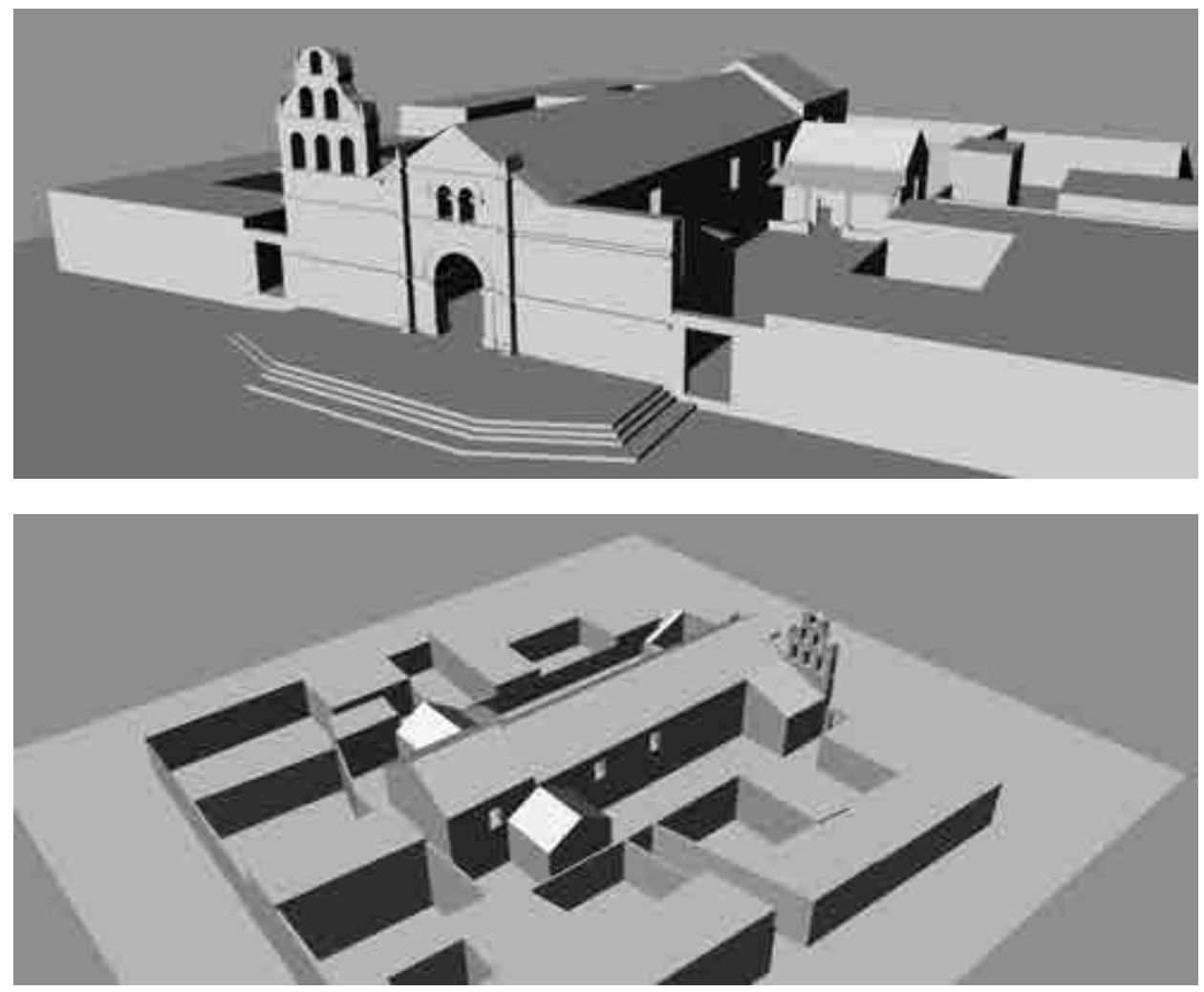

Figure 5 e 6. Modello $3 d$ della Chiesa e del suo intorno

Fonte: Elaborazioni grafiche degli autori.

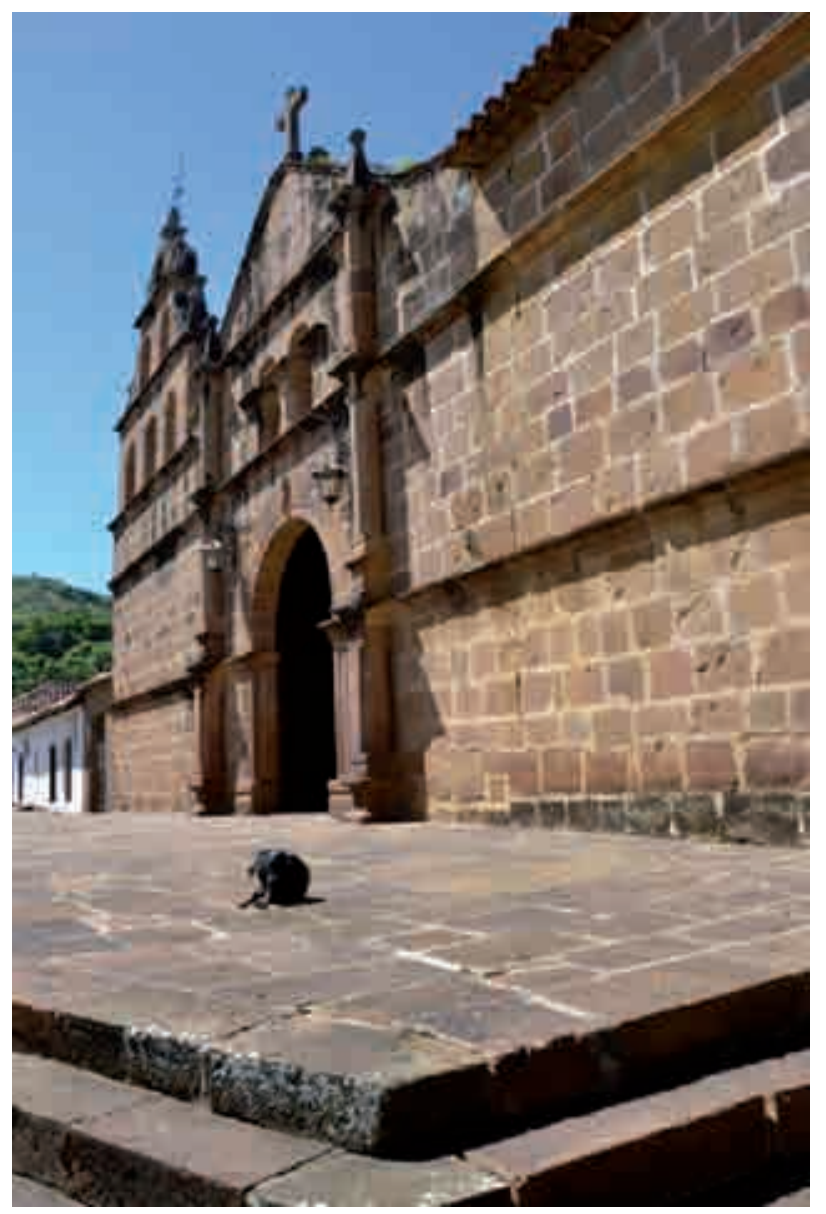

Figure 7. Facciata della Chiesa di Santa Lucia di Guane

Fonte: Archivio degli autori, 2013

M 15 REVISTA M VOL. 15 ENERO - DIIIEMBRE 2018 • FACULTAD DE ARQUITECTURA • UNIVERSIDAD SANTO TOMÁS COLOMBIA 
Figure 8. Retro della capella del Niño Jesús Fonte: Archivio degli autori, 2013.

Figure 9. Copertura

Fonte: Archivio degli autori, 2013.

Figure 10. Portico interno

Fonte: Archivio degli autori, 2013.
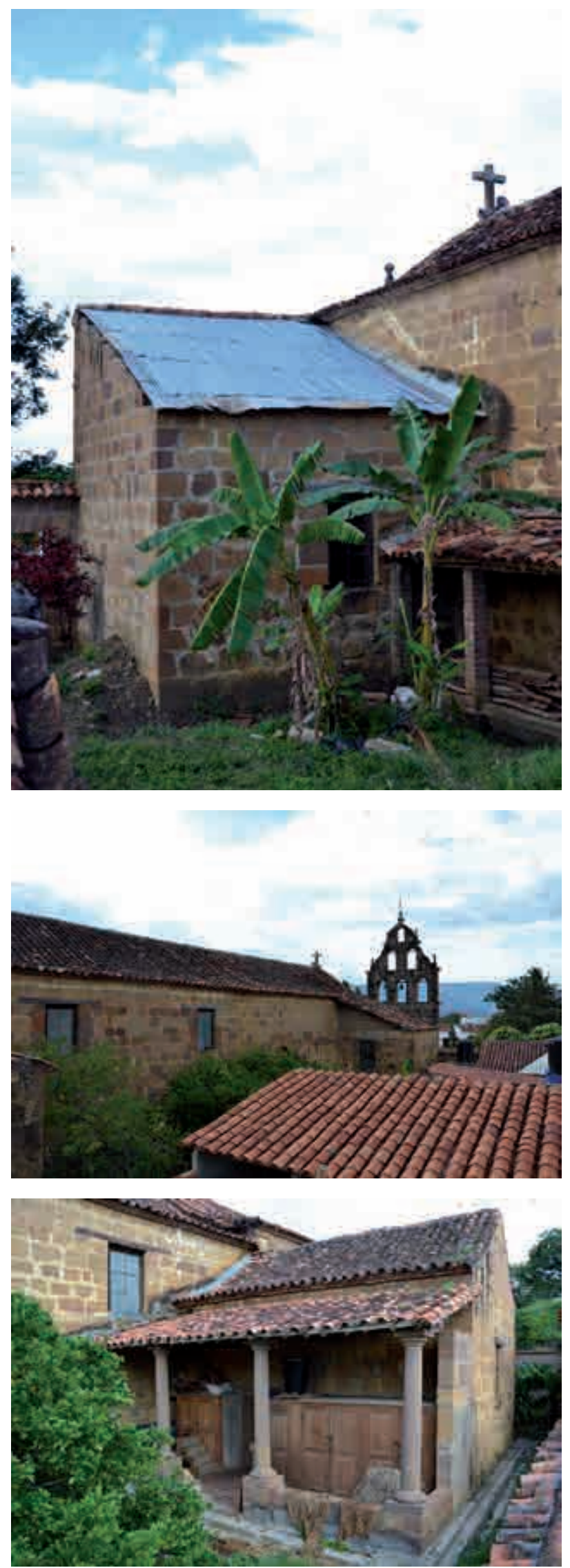
A scapito di ciò è importante tener conto dei diversi tipi di sollecitazione ai quali sono sottoposti i singoli elementi: i pares dovrebbero lavorare essenzialmente a compressione ma nella realtà risultano pressoinflessi e, per la loro snellezza, anche soggetti a carico di punta; le travi di bordo, invece, lavorano a flessione (Fig. I I).

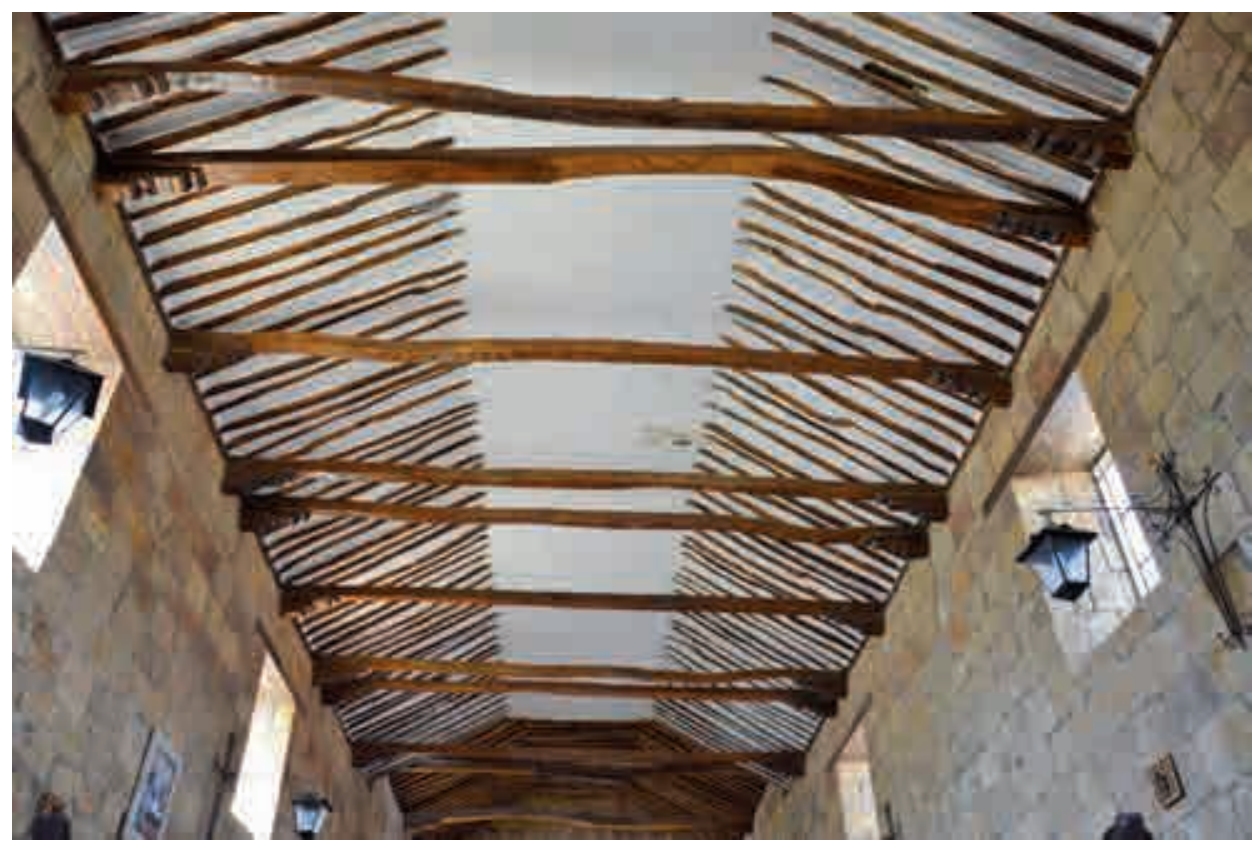

Per intervenire su un manufatto artistico ed elaborare un progetto di intervento conservativo bisogna, innanzitutto, essere a conoscenza della situazione materica, delle patologie di degrado delle quali soffre il monumento e dell'entità di queste.

Le problematiche legate all'alterazione sia fisiologica, sia patologica dei materiali, presentano generalmente cause e meccanismi similari, ma si distinguono per l'entità degli effetti. Ogni causa di degrado si presenta spesso come la somma, il frutto dell'interazione di più azioni di degrado prodotte da uno o più agenti naturali o antropici (Francesci, Germani, 2012). Possiamo definire cause intrinseche di degrado quelle legate alle caratteristiche del manufatto stesso, come errori progettuali, mentre sono definite estrinseche quelle caratteristiche dovute a fattori esterni verificatesi successivamente alla costruzione del manufatto e indipendenti da esso, fattori naturali improvvisi o prolungati nel tempo (Francesci, Germani, 20 I2). Per ogni prospetto è stato individuato il tipo di degrado materico, la possibile causa e le procedure di intervento per risolvere la patologia (Fig. 12).

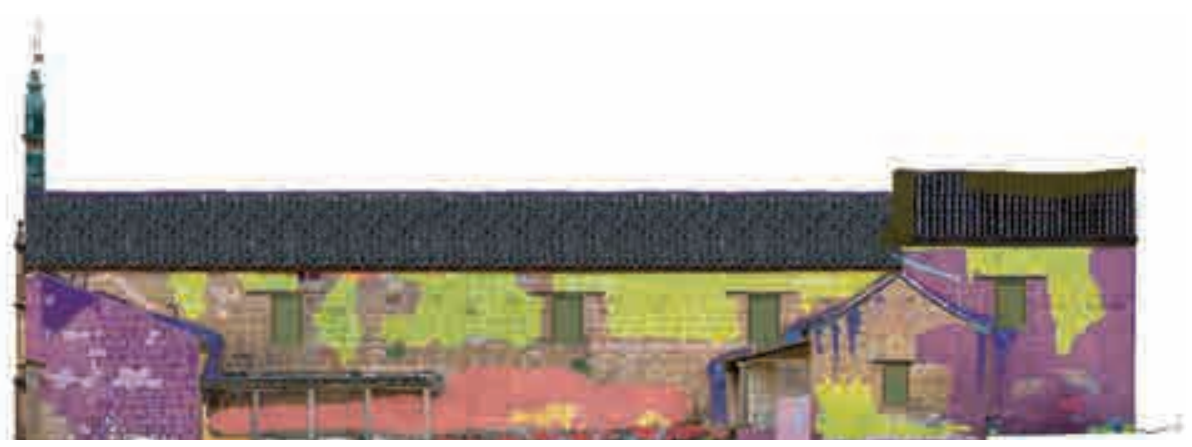

Figure II. Struttura del tetto Fonte: Archivio degli autori, 2012.
Figure I2. Fotopiano con le patologie di degrado Fonte: Elaborazione grafica degli autori, 2012. 
Il deterioramento dei materiali è un processo naturale, progressivo e praticamente inarrestabile, la cui conseguenza è la progressiva perdita delle qualità meccaniche del materiale. I danni biologici di natura essenzialmente estetica (alterazioni cromatiche, sviluppi di patine o presenza di vegetazione) vengono soventemessi più frequentemente in luce anche se in molti casi rappresentano l'aspetto meno importante del problema (Francesci, Germani, 20I2). Ogni indagine finalizzata allo studio del comportamento statico di una struttura con segni di sofferenza deve iniziare da una classificazione delle lesioni osservabili. Le fessure, essendo generate dal superamento della resistenza a trazione della pietra, possono indurre ad indirizzare correttamente le indagini. L'esame dei paramenti murari, nonché la valutazione sperimentale delle loro caratteristiche meccaniche, si prefigge di stabilire se la muratura in esame è capace di un comportamento strutturale idoneo a sostenere le azioni statiche e dinamiche previste e prevedibili per l'edificio in oggetto. II rilievo del quadro fessurativo consiste nell'acquisizione di tutti i sintomi di comportamento statico anomalo, al fine di risalire dagli effetti alle cause che li hanno determinati. Al fine di conseguire detta comprensione è necessario valutare i meccanismi di dissesto all'interno dell'ambito culturale e storico della fabbrica, secondo i materiali del luogo e secondo le caratteristiche architettoniche tradizionali. Nel quadro fessurativo della chiesa di Santa Lucia è stata fatta una distinzione tra le lesioni concentrate e quelle diffuse, le lesioni vecchie e quelle di nuova formazione, quelle da dissesto e quelle da assestamento. Si è rivelato particolarmente importante accertare se dette lesioni siano passanti o superficiali e se esista una netta corrispondenza tra le fessure sulle facce contrapposte dei muri.Le principali lesioni rilevate sull'edificio sono provocate da sforzi di taglio, causate da trazione verticale o orizzontale. Le prime, quelle di taglio verticale, sono di norma dovute a cedimenti differenziali delle fondazioni o ad azioni sismiche, le seconde, quelle di taglio orizzontale, sono determinati più che altro da movimenti di rotazione e si manifestano con andamento diagonale. Un' ultima tipologia di lesioni che interessa la chiesa di Santa Lucia è quella che possiamo definire di assestamento, si presenta con andamento principalmente verticale ed evidenti soluzioni di continuità. La facciata dell'edificio presenta una concentrazione di lesioni, gravi e passanti, nella parte destra, in corrispondenza della congiunzione del corpo della navata con la cappella del Niño Jesús. Notiamo una frattura che percorre verticalmente l'intero paramento murariocon andamento a gola rovescia con un'ampiezza che va dai 40 ai $60 \mathrm{~mm}$ con profondità di $58 \mathrm{~cm}$ (Fig. I3, I4, I5, I6).

Lo stesso andamento a gola rovescia, ma sviluppato in maniera speculare rispetto a quello prima descritto, si ritrova all'interno della cappella. Questo è sintomo di un movimento di rotazione o traslazione verticale non uniforme, dovuta al cinematismo di distacco che sta subendo la suddetta cappella. Le cause principali possono essere riscontrate nella scarsa profondità della fondazione (sono stati fatti dei saggi dai quali è risultata una fondazione di soli $40 \mathrm{~cm}$ su questo lato) e nel cattivo drenaggio del terreno, che non risulta in grado di restituire alla struttura una spinta uguale e contraria al proprio peso. II cattivo ammorsamento della cappella alla navata e l'apparecchiatura muraria a sacco vanno ad incrementare ulteriormente le cause del dissesto (Fig. I7).

Oltre alle sopra citate lesioni che riguardano la facciata e la parete nord della cappella del Niño Jesús, vi sono altre importanti fratture presenti sul paramento murario di questa. Nella parete ovest risulta evidente il proseguimento del movimento di collasso rotazionale dell'elemento in direzione dell'angolo nord con la creazione di quelle lesioni tipiche dei cedimenti terminale nei muri ad angolo. Particolarmente delicata si presenta la situazione della parete posteriore della cappella destra, nella zona di attacco col corpo della nave centrale; una larga parte di paramento a partire dallo stipite, ha subito un completo distacco dall'apparecchiatura muraria e, se non fosse per il portichetto laterale che senza volerlo svolge un ruolo di puntone frenandone il cinematismo, sarebbe probabilmente già collassata. La maggiore lesione di tutto il manufatto architettonico presenta un ventre nella parte sommitale di $20 \mathrm{~cm}$. (Fig. 19). 

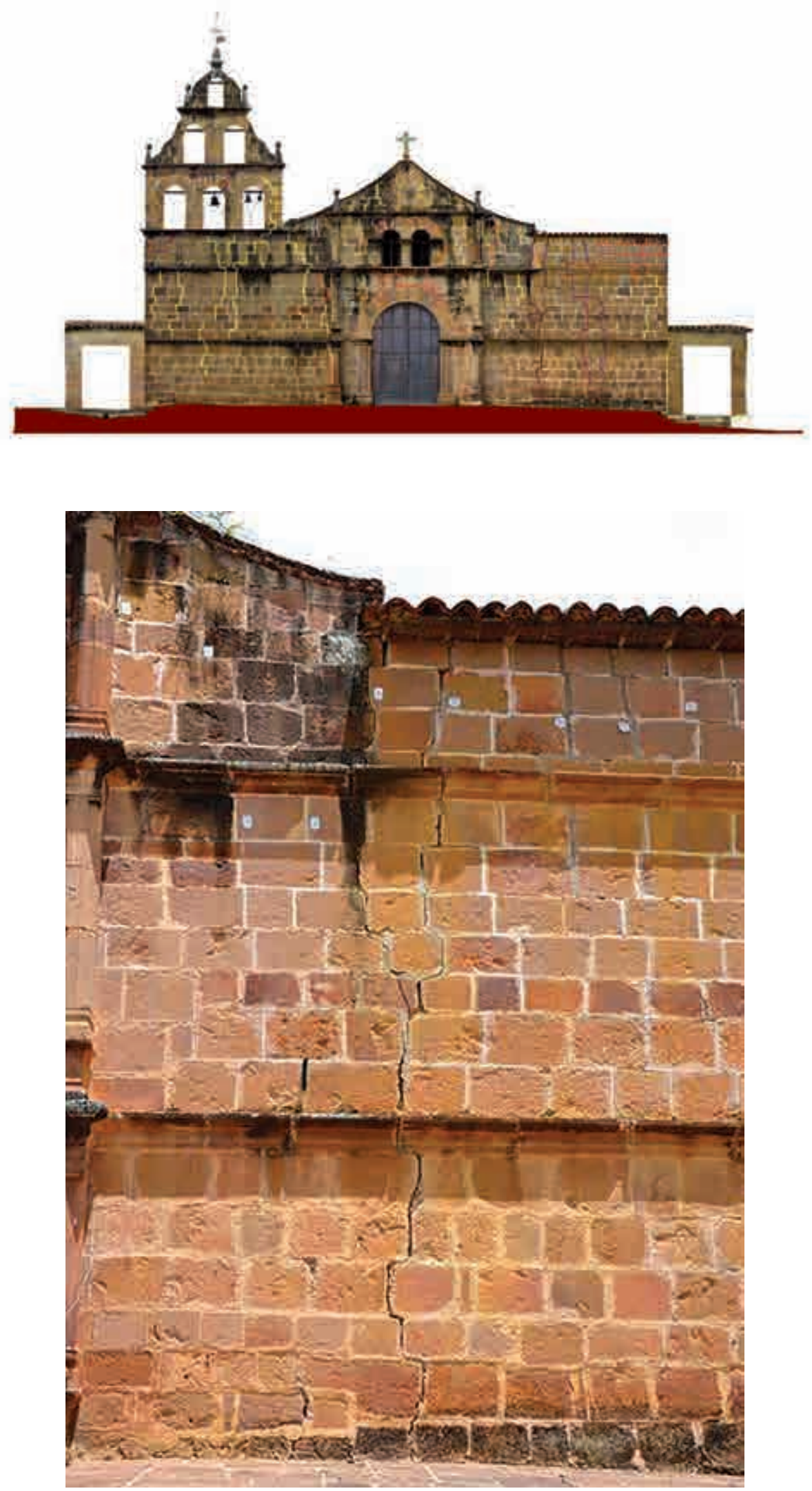

Figure 13. Quadro fessurativo della facciata della chiesa di Santa Lucia di Guane

Fonte: Elaborazione grafica degli autori, 2012.
Figure 14. Distacco della cappella del Niño Jesús

Fonte: Archivio degli autori, 2012. 
Figure I5. Ediotipo del rilievo delle lesioni in facciata

Fonte: Elaborazione grafica degli autori, 2012.

Figure 16. Ampiezza delle lesioni in facciata (2012)

Fonte: Archivio degli autori, 2012.

Figure 17. Ipotesi sulla apparecchiatura delle murature portanti

Fonte: Elaborazione grafica degli autori, 2012

\section{Lesione $30-31$}
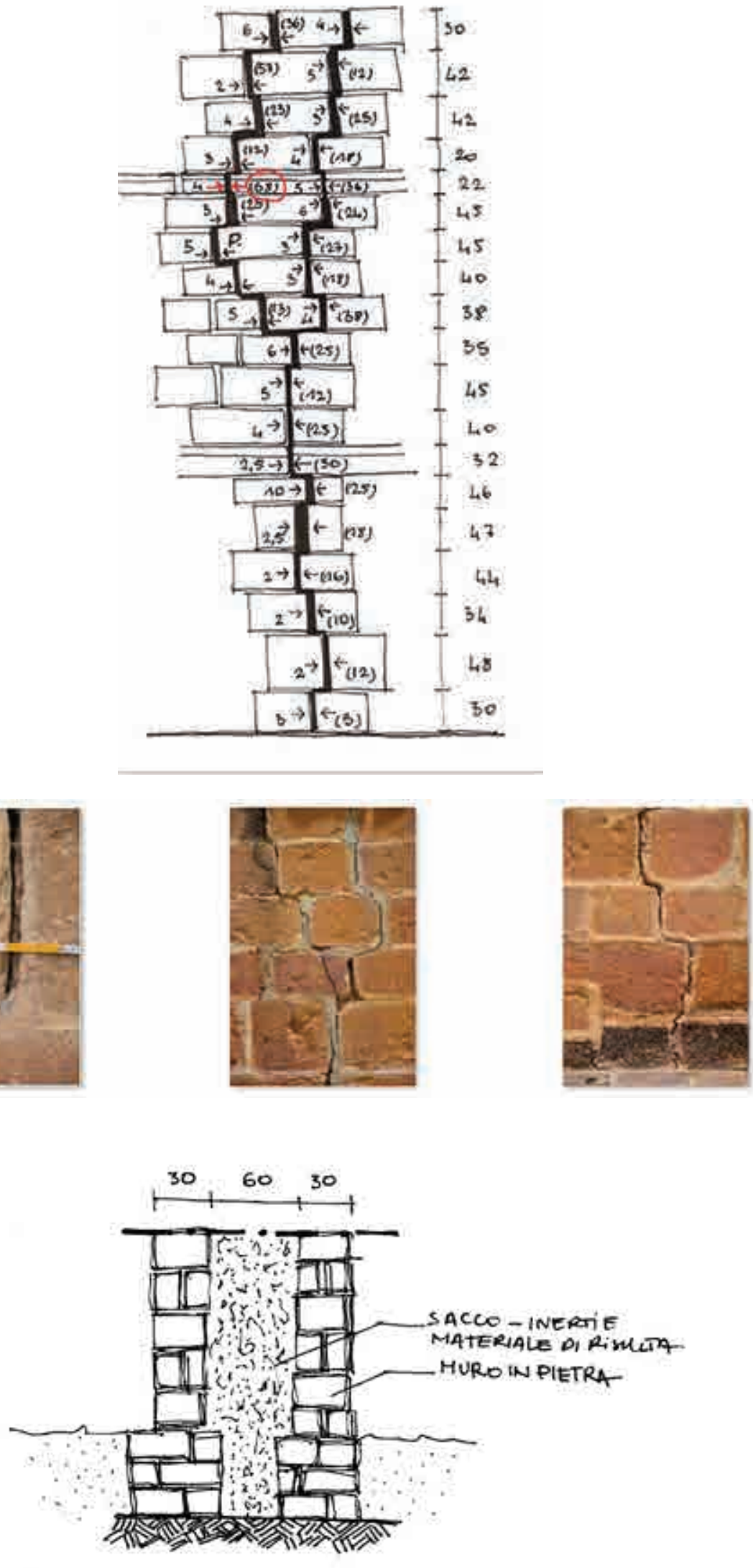

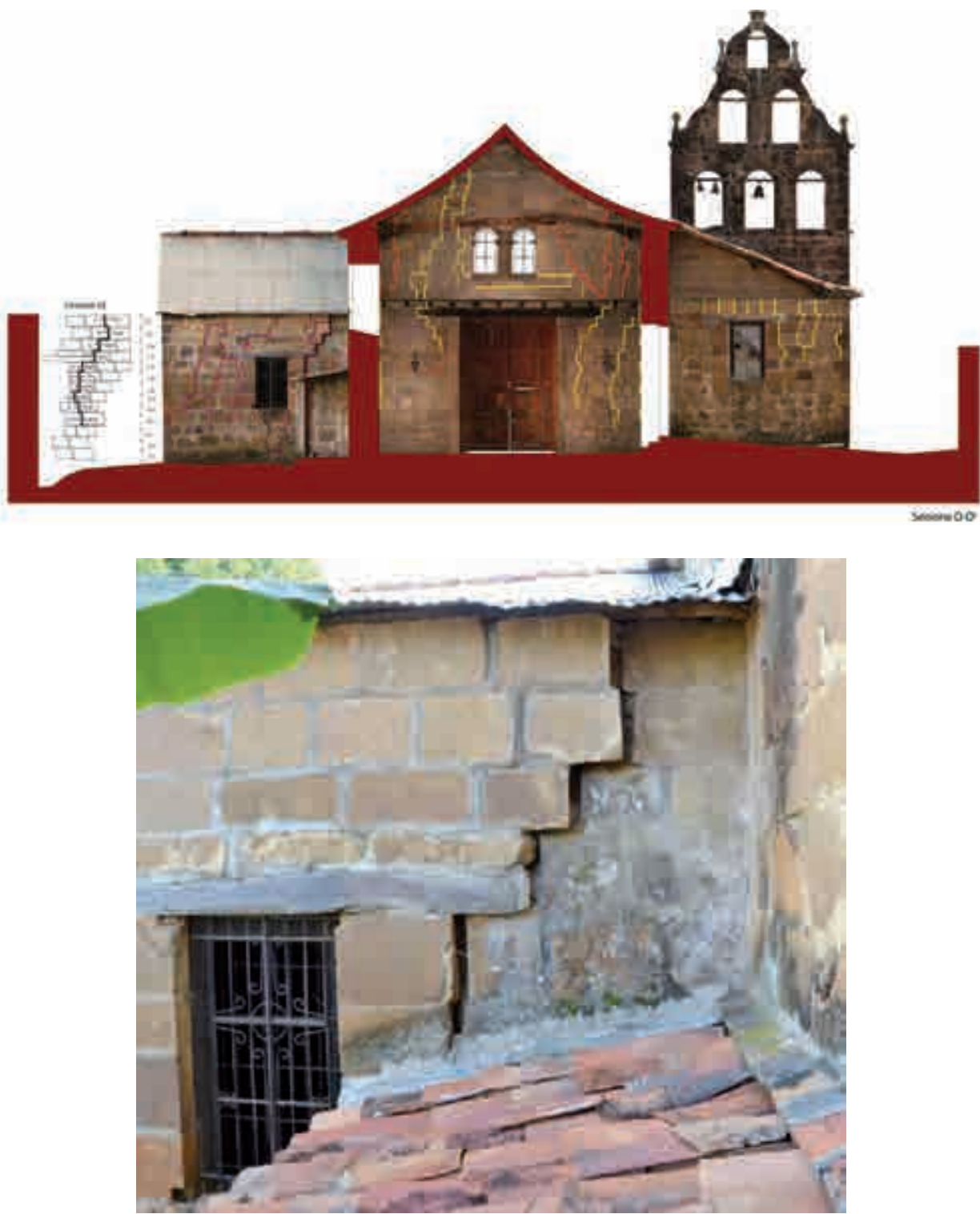

Analizzando la cappella del Niño Jesús da un punto di vista globale, notiamo che queste lesioni fanno parte di un unico movimento che tende a distaccarla dal corpo centrale, movimento confermato anche dal fuori piombo registrato durante la fase di rilievo: $12 \mathrm{~cm}$ su punto centrale della parete e $8 \mathrm{~cm}$ agli angoli.

Per consentirci di formulare un giudizio diagnostico articolato ed organico sono state effettuate prove fisiche ed esami chimici su campioni di materiale raccolto. Sono state effettuate prove di resistenza a compressione su campioni di pietra arenaria provenienti dalla muratura della chiesa, posizionandoli nell'apposito strumento; appoggiandolo sul piatto inferiore e collocando i quattro sensori che registrano le deformazioni ognuno ad un angolo della piastra superiore. La pressione è stata applicata gradualmente fino alla completa rottura del provino. Attraverso la prova di resistenza a compressionesiamo in grado di ottenere il diagramma tensioni-deformazioni. Abbiamo riscontrato buone caratteristiche meccaniche (resistenza a compressione c.a. $5 \mathrm{Kg} / \mathrm{mmq}$ ) ma, come tutte le arenarie, il materiale perde velocemente le sue proprietà dato che si tratta di una pietra fortemente porosa e di conseguenza facilmente aggredibile dagli agenti atmosferici.Successivamente si è modellata l'intera
Figure 18. Quadro fessurativo della facciata posteriore

Fonte: Elaborazione grafica degli autori, 2012.
Figure 19. Disammorsamento nello spessoredella parete posteriore della cappella del Niño Jesús

Fonte: Archivio degli autori, 2012. 
Figure 20. Stato tensionale. Le zone in rosso indicano i massimi livelli di trazione Fonte: Elaborazione grafica degli autori.

Figure 21. Stato tensionale per cedimenti impressi

Fonte: Elaborazione grafica degli autori. struttura della chiesa con il codice di calcolo agli elementi finiti STRAUS7, che, sperimentato lungamente in varie occasioni di studio, ha dato prova di ottime performances anche su edifici storici in muratura. II codice lavora su un modello continuo elastico e isotropo, l'intera chiesa è stata modellata e discretizzata con elementi finiti di tipo "brick" e, parzialmente con elementi di tipo "plate". Va però detto che, assumendo il codice un modello di comportamento elastico-lineare, che non corrisponde al reale modello della muratura (unilatero con limitazione sul segno delle tensioni) i risultati della analisi numerica devono essere considerati solo come capaci di fornire il quadro delle zone di intrinseca debolezza della struttura, quadro che poi va comparato con il reale stato fessurativo (Gallo, 2007). L'analisi è stata condotta con soli pesi propri e, verificata la buona risposta della struttura a questi, successivamente si è proceduto ad applicare oltre al peso proprio, un'azione distorcente di tipo cinematico (cedimento verticale) alla base destra della facciata, da noi ipotizzato come principale punto debole dell'intera chiesa (Fig. 20). Si può affermare che lo stato tensionale e deformativo ipotizzato è compatibile con il quadro fessurativo reale (Gallo, 2007). Molto probabilmente ciò che si è verificato è l'innescarsi del cedimento nella parete est della cappella del Niño Jesús, per poi propagarsi, con andamento semi lineare, alle circostanti pareti (Fig. 20, 2I).
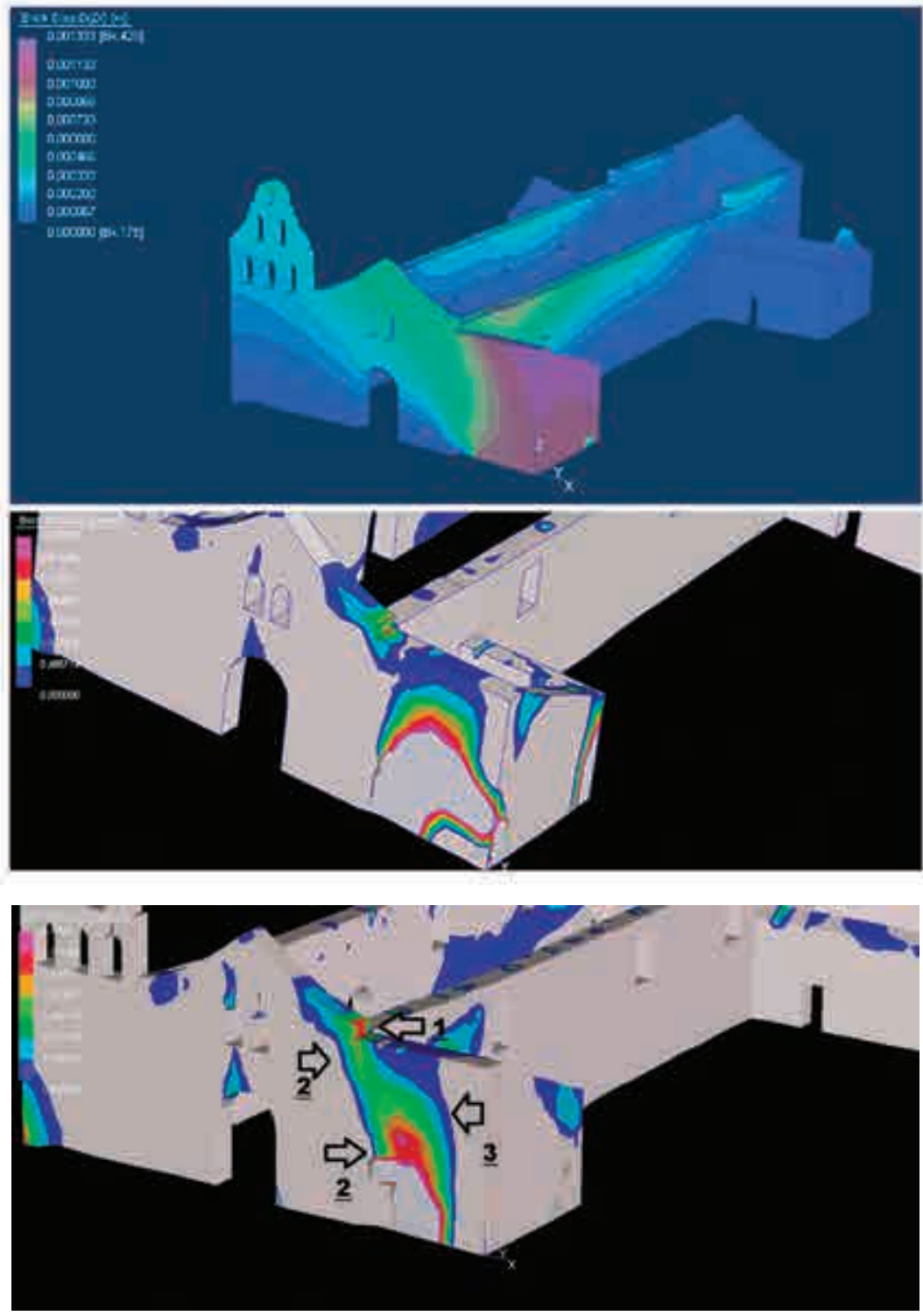


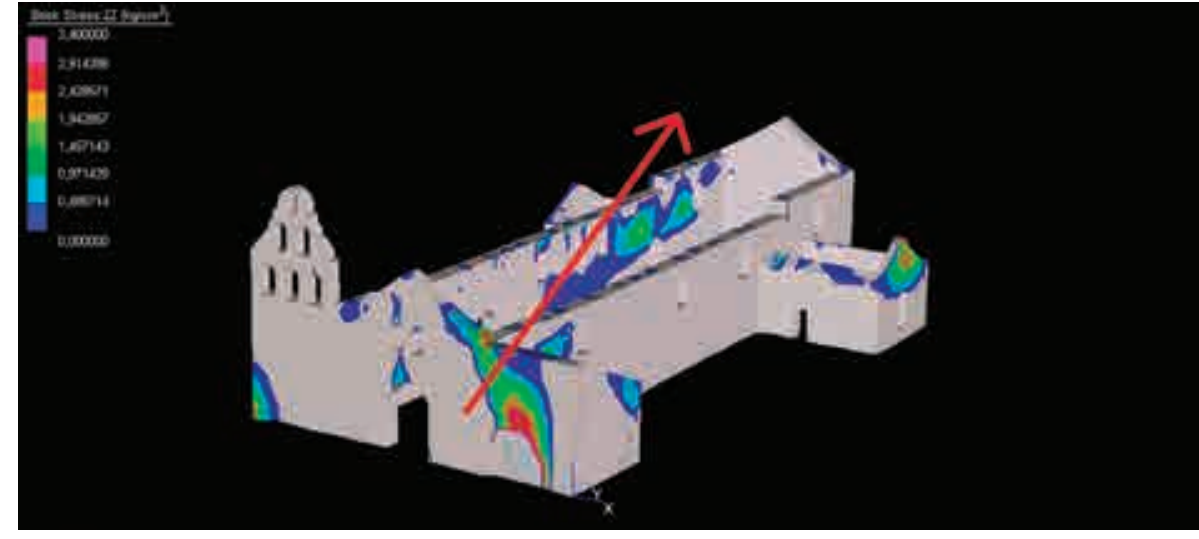

In conclusione la probabile causa del dissesto della chiesa di Santa Lucia a Guane è da ascriversi, in parte, alla presenza di fondazioni di scarsa profondità e consistenza geometrica. Ciò parrebbe aver portato al già citato cinematismo dell'angolo estremo destro della facciata, provocando il distacco del corpo della cappella. II suolo sul quale appoggia l'edificio risulta essere di tipo sabbioso-argilloso e presenta quindi caratteristiche di terreno espansivo che assorbe e trattiene l'acqua piovana, andando a generare movimenti di dilatazione e compressione nel terreno che si ripercuotono sull'edificio. I cedimenti differenziali del suolo portano a stati di tensione interna ai materiali ai quali la struttura non è in grado di resistere (Mastrodicasa, 1974). Attraverso la formazione di lesioni abbiamo una redistribuzione delle azioni mutue terreno-struttura, la quale, da una parte è causa di dissesti, ma dall'altra ha un effetto stabilizzante. Sulla base dello studio effettuato nel 2012 , si ipotizzarono interventi di consolidamento, con un approccio di totale compatibilità meccanica tra i materiali presenti e quelli costituenti il consolidamento. In sintesi venne proposta la realizzazione di un nuovo sistema in subsuolo superficiale di defluimento delle acque piovane, in maniera da allontanarle il più possibile dal corpo di fabbrica (tenere presente che la parte tergale della chiesa poggia in fondazione ad una quota tre metri più alta del centro della piazza antistante). Venne progettata una rilivellazione della base fondale, con il metodo della sottomurazione ed usando la stessa pietra caliza, che regolarizzasse il piano fondale (in pendenza) e lo approfondisse molto oltre gli attuali 40-60 cm. Venne ancora proposta la realizzazione, perimetralmente alla chiesa, di uno scannafosso (trincea) sempre allo scopo di proteggere le fondazioni dalla imbibizione per capillarità, In attesa dei relativi finanziamenti per la completa messa in sicurezza, si propose un adeguato sistema di puntellamento ligneo (Gallo, 2007) (Fig. 23, 24).

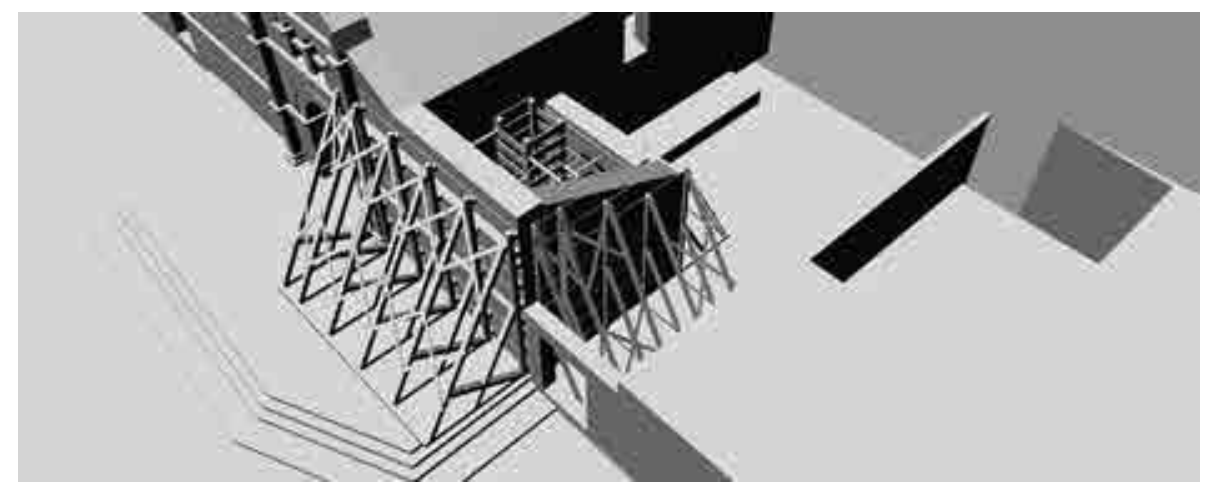

Figure 22. Direzione di propagazione delle lesioni dovute al cedimento fondazionale Fonte: Elaborazione grafica degli autori.
Figure 23. Sistema di puntellamento proposto per la cappella in cedimento Fonte: Elaborazione degli autori. 


\section{FASCIA B}

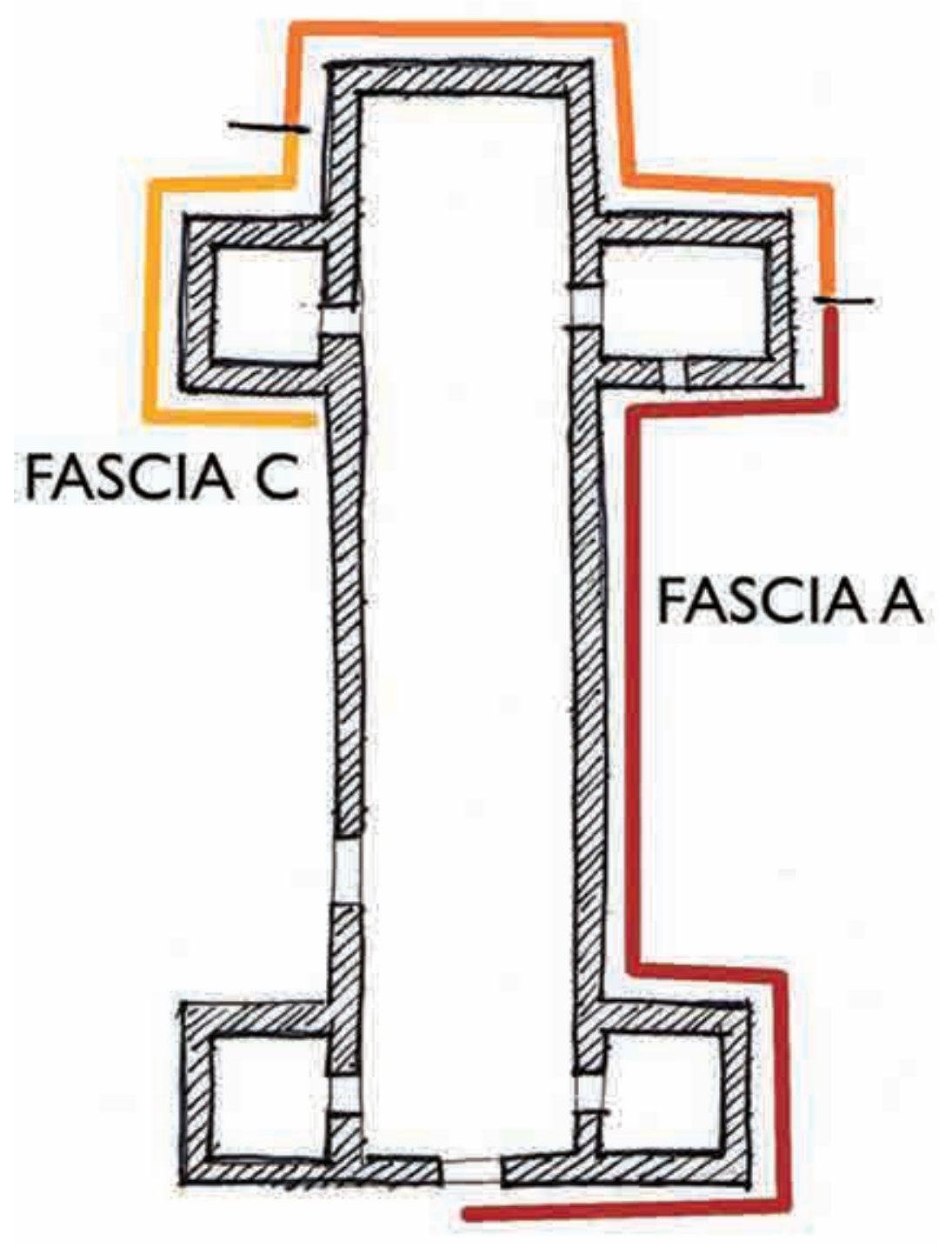

\section{CONCLUSIONI}

Dal 2013 ad oggi, nonostante lo studio fosse stato concluso, non è stata mai abbassata la guardia sia da parte dell’Università Santo Tomás, sia da parte del Dipartimento fiorentino, sollecitando azioni ai vari Sindaci che si sono succeduti nella città di Barichara, da cui Guane dipende, lo stesso per i Governatori di Santander, il Ministero della Cultura e naturalmente, la Chiesa Cattolica, nonostante siano cambiati ben due parroci in Guane.

La ricerca, presentata in modo suscinto in questo articolo, lascia intravedere i diversi problemi che affligono la Chiesa di Santa Lucia, tra questi strutturali, il deterioramento dei materiali da costruzione e le rese del suolo. Inoltre, fornisce linee guida chiare per l'attivazione degli interventi necessari per contrastare queste patologie. 
Nonostante ciò, finora non è stato fatto nessun intervento di rilievo nemmeno per realizzare il puntello provvisionale delle aree che minacciano di crollare. Si parla di mancanza di soldi per avviare le relative procedure, anche se pare che fossero disponibili per la Chiesa, segregati da qualche parte, ben 40 milioni di pesos colombiani.

Purtroppo, la storia della Basilica Minore di Socorro si è ripetuta a Guane, con la stessa mancanza di dialogo costruttivo tra la Chiesa Cattolica e il Ministero della Cultura: di conseguenza, nella Chiesa di Santa Lucia, il tetto di una delle cappelle è crollato ed è stato ricostruito con un controsoffitto di materiale plastico chiaro e le svariate lesioni sono state semplicemente stuccate (Fig. 25).

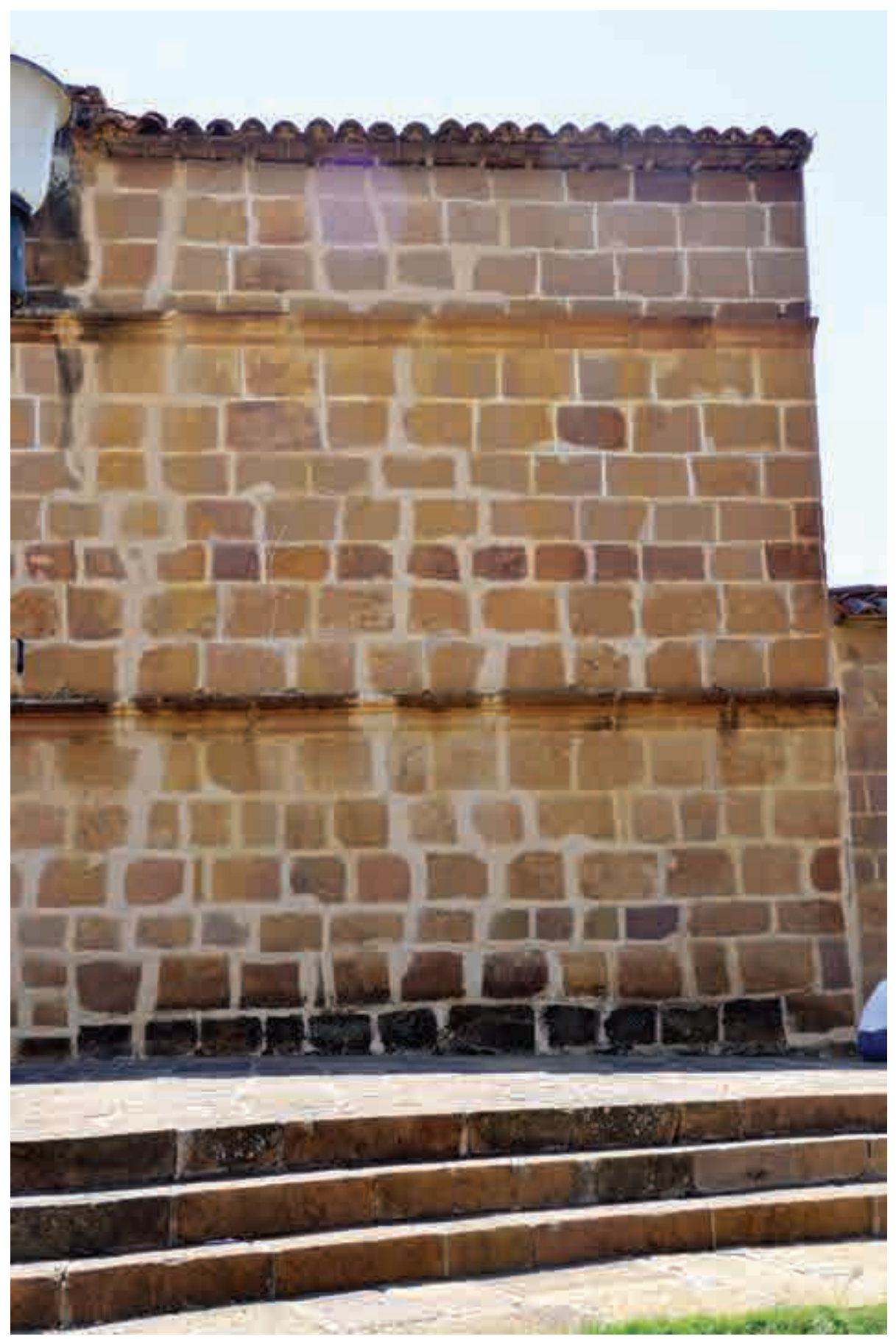


Solo che già si nota, a solo un anno e mezzo dal "riabbellimento" e non poteva che essere altrimenti, che le lesioni si stanno riaprendo. In altra sezione del documento abbiamo riflettuto sulle ragioni di questo incomprensibile atteggiamento generale, adducendone la colpa alla mancanza di volontà culturale e politica. Ma forse le ragioni stanno molto più nel profondo, e risalgono al fatto che per troppo tempo l'America Latina ha seguito modelli di sviluppo umano non propri.

Forse la ragione sta nel significato delle parole che qui seguono e che partono da un sentimento comune nei Paesi dell'America precolombiana, riferito alla gestazione degli antecedenti sull'intervento nel territorio: questi si formarono dalla confluenza di contingenti profondamente dispari riguardanti le loro caratteristiche razziali, culturali e linguistiche, come prodotto dei progetti coloniali europei: spagnolo, francese, tedesco, portoghese e italiano tra gli altri; chi promossero nell'America delle Ande un proposito di dominazione in cui la religione era la cornice di uno sviluppo diseguale, che imprissero profondamente nelle nostre coscienze una diminuzione del valori nostrani, lo sfruttamento eccessivo delle nostre risorse naturali e l'intricato e malevolo modo di fare politica. E così sembrerebbe che ci siamo persi nella memoria dei nostri antenati aborigeni, di voler apparire come chiuque altro i, tranne noi stessi.

\section{REFERENZE}

Ardila, I. (2005). El pueblo de los Guanes, raíz gloriosa de Santander. Bogotá: Instituto Colombiano de Cultura.

Arenas, E. (2004). Los guanes: el pueblo de la cingla. Ediciones Universidad Santo Tomás Seccional Bucaramanga.

Arenas, E. (2006). Siete lenguas: proceso histórico de poblamiento y posicionamiento urbano en Santander. Ediciones Universidad Santo Tomás Seccional Bucaramanga.

Ciaccia, P. (2003). Caratteri costruttivi dell'edilizia storica; diagnosi dei dissesti e tecniche di intervento. Napoli: Edizioni Sistemi Eitoriali.

Cigni, G. (1978). II consolidamento murario. Tecniche di intervento. Roma: Edizioni Kappa.

Defez, A. (1998). II consolidamento degli edifici. Napoli: Liguori editore.

Francesci, S., Germani, L. (20I2). II degrado dei materiali nell'edilizia. Roma: Edizioni Dei-Tipografia del Genio Civile.

Gallo, A. (2007). Sul consolidamento degli edifici storici. Roma: Edizioni EPC Libri. 
Ibañez, C. (2004). Caracterización sociocultural del municipio de Barichara y su entorno. Ediciones Fundación José María Delgado.

Mastrodicasa, S. (1974). Dissesto statici delle strutture edilizie: diagnosi e consolidamento. Edizioni Ulrico Hoepli Milano.

Pbro. Carreño, D. (2010). Proceso de evangelización de las provincias del sur de Santander durante la Colonia y el siglo XIX (prima edizione, 1998). Ediciones Fundación Universitaria San Gil.

Ribero, H. (1986). Un recorrido por la provincia de los indígenas Guanes del departamento de Santander: desde la época prehispánica hasta su desaparición. Bucaramanga: SIC.

Silvarangel, H. (200I). Retazos históricos de mi pueblo Barichara. Comulseb.

Todopatrimonio.com (2018). Cartas y convenciones. Recuperado de http://todopatrimonio.com/cartas-y-convenciones/

M 27 REVISTA M VOL. 15 ENERO - DICIEMBRE 2018 • FACULTAD DE ARQUITECTURA • UNIVERSIDAD SANTO TOMÁS COLOMBIA 